$50^{t h}$ AIAA Aerospace Sciences Meeting and Exhibit, 9-12 January 2012, Nashville, Tennessee.

\title{
Dynamics of Unsteady Inviscid and Viscous Detonations in Hydrogen-Air
}

\author{
Christopher M. Romick* \\ University of Notre Dame, Notre Dame, Indiana, 46556-5637, USA \\ Tariq D. Aslam ${ }^{\dagger}$ \\ Los Alamos National Laboratory, Los Alamos, New Mexico 87545, USA \\ Joseph M. Powers ${ }^{\ddagger}$ \\ University of Notre Dame, Notre Dame, Indiana, 46556-5637, USA
}

\begin{abstract}
The dynamics of one-dimensional, overdriven, hydrogen-air detonations predicted in the inviscid limit as well as with the inclusion of mass, momentum, and energy diffusion were investigated. A series of shock-fitted calculations was performed in which the overdrive was varied in the inviscid limit. The $0.97 \mathrm{MHz}$ frequency of oscillation predicted for a $f=1.1$ overdriven detonation agrees well with the value of $1.04 \mathrm{MHz}$ observed by Lehr in the equivalent shock-induced combustion experiment around a spherical projectile. As the initial overdrive is lowered, the long time behavior of the system becomes more complex, causing the amplitude of pulsations to increase and oscillations at multiple frequencies to appear. When the viscous analog of these detonations was simulated, it was found that viscous effects slightly alter the structure of a stable detonation and can significantly decrease the amplitude of pulsations in an unstable detonation.
\end{abstract}

\section{Introduction}

A detonation is shock-induced combustion wave in which exothermic energy release contributes to driving the shock. This exothermic energy release is a consequence of chemical reactions, and these reactions occur on various scales. As demonstrated by Powers and Paolucci ${ }^{1}$ using a spatial eigenvalue analysis on a hydrogenair model, for a steadily traveling Chapman-Jouguet (CJ) detonation with an ambient state at standard atmospheric conditions, these scales range from $10^{-5} \mathrm{~cm}$ to $10^{0} \mathrm{~cm}$ at equilibrium; away from equilibrium the breadth of scales can be even larger. These fine reaction scales are a manifestation of an averaged representation of the molecular collision model in which the fundamental length scale is the mean free path, Al-Khateeb et al. ${ }^{2}$ In order to have a mathematically verified prediction, this wide range of scales must be resolved; as such, studies of detonations using detailed kinetics models have been limited.

As Shepherd ${ }^{3}$ stated in his review, much of the modeling of detonations has been done with simplified kinetics in the inviscid limit. However, there have been some studies done with detailed kinetics. One of the first was by Sussman, ${ }^{4}$ who performed one-dimensional, unsteady simulations of hydrogen-air detonations in the inviscid limit using only 20 points in the induction zone with an ambient pressure of $0.421 \mathrm{~atm}$. Using a massively parallel computing environment, Oran et al. ${ }^{5}$ performed an inviscid study of the development of detonation cells in two dimensions at an ambient pressure of $6.58 \times 10^{-2}$ atm in a hydrogen-oxygenargon mixture. While studying a hydrogen-oxygen mixture diluted by argon in the inviscid limit, Eckett ${ }^{6}$ found that a minimum of 150 points in the induction zone was necessary to capture the dynamics of an overdriven, inviscid detonation for a mixture at an ambient atmospheric pressure. Using an adaptive method, Singh et al. ${ }^{7}$ simulated a one-dimensional, unsteady, viscous detonation in a hydrogen-oxygen-argon mixture.

*Ph.D. Candidate, Department of Aerospace and Mechanical Engineering, and AIAA Student Member. cromick@nd.edu

$\dagger$ Technical Staff Member, Dynamic and Energetic Materials Division, and AIAA Member.

$\ddagger$ Professor, Department of Aerospace and Mechanical Engineering, and AIAA Associate Fellow. 
Yungster and Radhakrishan ${ }^{8}$ performed a convergence study and found that for a hydrogen-air mixture with an ambient pressure of $0.197 \mathrm{~atm}$ a minimum resolution of near one micron was necessary to capture the dynamics of the detonation in the inviscid limit with shock-capturing. Studying the same highly dilute mixture as Oran et al., Hu et al. ${ }^{9,10}$ suggested that in order to accurately capture the dynamics of the twodimensional, inviscid, unsteady detonation, a minimum resolution of 25 microns or 440 points in the induction zone was necessary. Daimon and Matsuo ${ }^{11}$ examined various one-dimensional, inviscid, overdriven, hydrogenair detonations and found that as the overdrive was decreased, the long time behavior of the detonation became more complex, similar to the results found by Sussman, Eckett, and Yungster and Radhakrishan for other hydrogen-based mixtures. While employing an adaptive mesh in a parallel computing environment, Ziegler et al. ${ }^{12}$ examined a viscous, double Mach reflection detonation in a hydrogen-air mixture and found that even with a finest resolution near a micron that their results only exhibited qualitative convergence.

It is clear that the majority of the work performed modeling detonations has been done in the inviscid limit, as it is sometimes thought that viscous effects are small in detonations or other high-speed flows. However, this assumption of neglecting viscous effects may be problematic. In detonations propagating into atmospheric ambient mixtures there are reaction dynamics and steep gradients near a micron scale suggesting that physical diffusion could play an important role. In fact, while using resolutions near a micron in a three-dimensional inviscid simulation to predict the behavior of spinning detonations, Tsuboi et al. ${ }^{13}$ report that while some structures appear to converge, "The present results cannot resolve such cross-hatchings in the ribbon because of a lack of grid resolution." Deiterding ${ }^{14}$ also reported that the interactions between chemistry and hydrodynamic flow in inviscid detonations "in general exhibit a strong dependency on the mesh spacing." In a two-dimensional study of detonation patterns, Powers ${ }^{15}$ found that patterns were strongly grid-dependent for the reactive Euler equations, but for the reactive Navier-Stokes equations relaxed to a grid-independent dissipative structure. This suggests that numerical diffusion is playing a significant role. Therefore, one should consider the introduction of grid-independent physical diffusion. We have previously investigated detonations with the addition of mass, momentum, and energy diffusion to the model in the onestep model. ${ }^{16}$ It was shown that when the viscous length scale is similar to that of the finest reaction scale, viscous effects play a critical role in determining the long time behavior of the detonation. In particular, viscous effects delay the transition to instability, and can play a dramatic role for detonations in the highly nonlinear regime characterized by oscillations at multiple frequencies.

The goal of this paper is to explore the long time dynamics of a hydrogen-air detonation using detailed kinetics in the inviscid limit and to demonstrate how the addition of physical mass, momentum, and energy diffusion affects the system. The plan of this paper is as follows. The mathematical model is presented followed by a description of the computational methods. This model is used to predict the dynamics of several overdriven hydrogen-air detonations. It will be shown that as the initial overdrive is decreased, the long time behavior becomes more complex, in agreement with similar previous studies performed in the inviscid limit. As seen in the one-step model, the addition of viscous terms will be shown to have a stabilizing effect.

\section{Model}

\section{A. Mathematical model}

The governing equations are the reactive, compressible Navier-Stokes equations and are expressed as

$$
\begin{aligned}
\frac{\partial \rho}{\partial t}+\nabla \cdot(\rho \mathbf{u}) & =0 \\
\frac{\partial}{\partial t}(\rho \mathbf{u})+\nabla \cdot(\rho \mathbf{u u}+p \mathbf{I}-\boldsymbol{\tau}) & =\mathbf{0}, \\
\frac{\partial}{\partial t}\left(\rho\left(e+\frac{\mathbf{u} \cdot \mathbf{u}}{2}\right)\right)+\nabla \cdot\left(\rho \mathbf{u}\left(e+\frac{\mathbf{u} \cdot \mathbf{u}}{2}\right)+(p \mathbf{I}-\boldsymbol{\tau}) \cdot \mathbf{u}+\mathbf{q}\right) & =0 \\
\frac{\partial}{\partial t}\left(\rho Y_{i}\right)+\nabla \cdot\left(\rho \mathbf{u} Y_{i}+\mathbf{j}_{i}\right) & =\bar{M}_{i} \dot{\omega}_{i},
\end{aligned}
$$

where Eqs. (11)-(41) represent the conservation of mass, linear momenta, energy and the evolution of species. The independent variables are $t$, the temporal coordinate, and $\mathbf{x}$ the spatial coordinates. The dependent variables are the mixture mass density, $\rho$, the mixture velocity components, $\mathbf{u}$, the mixture pressure, $p$, the viscous stress tensor, $\boldsymbol{\tau}$, the specific internal energy of the mixture, $e$, the total heat flux vector, $\mathbf{q}$, the mass fraction, $Y_{i}$, the diffusive mass flux, $\mathbf{j}_{i}$, the molecular mass, $\bar{M}_{i}$, and the molar production rate per 
unit volume, $\dot{\omega}_{i}$, for the $i^{\text {th }}$ specie. The $\nabla$ symbol is the gradient operator, and $\mathbf{I}$ is the identity matrix. Equations (11) and (3) are scalar equations, and Eqs. (2) and (4) vector equations of lengths $d$ and $N-1$, respectively, where $\mathrm{d}$ is the dimension of the problem, and $N$ is the number of species. To close the system, constitutive relations must be specified; the following constitutive relations have been chosen for a mixture of $N$ species composed of $L$ elements interacting in $J$ reactions:

$$
\begin{aligned}
& \mathbf{j}_{i}=\rho \sum_{\substack{k=1 \\
k \neq i}}^{N} \frac{\overline{M_{i}} D_{i k} Y_{k}}{\bar{M}}\left(\frac{\nabla y_{k}}{y_{k}}+\left(1-\frac{\overline{M_{k}}}{\bar{M}}\right) \frac{\nabla p}{p}\right)-\frac{D_{i}^{T} \nabla T}{T}, \\
& \boldsymbol{\tau}=\mu\left(\nabla \mathbf{u}+(\nabla \mathbf{u})^{T}-\frac{2}{3}(\nabla \cdot \mathbf{u}) \mathbf{I}\right), \\
& \mathbf{q}=-\mathrm{k} \nabla T+\sum_{i=1}^{N} \mathbf{j}_{i} h_{i}-\mathcal{R} T \sum_{i=1}^{N} \frac{D_{i}^{T}}{\overline{M_{i}}}\left(\frac{\nabla y_{i}}{y_{i}}+\left(1-\frac{\overline{M_{i}}}{\bar{M}}\right) \frac{\nabla p}{p}\right), \\
& y_{i}=\frac{\bar{M}}{\overline{M_{i}}} Y_{i} \\
& \bar{M}=\left(\sum_{i=1}^{N} \frac{Y_{i}}{\overline{M_{i}}}\right)^{-1} \\
& p=\rho R T \text {, } \\
& R=\frac{\mathcal{R}}{\bar{M}} \text {, } \\
& h_{i}=h_{i}^{r e f}+\int_{T_{r e f}}^{T} c_{P, i}(\hat{T}) d \hat{T} \\
& e=\sum_{i=1}^{N} Y_{i}\left(h_{i}-\frac{\mathcal{R} T}{\bar{M}_{i}}\right) \\
& \dot{\omega}_{i}=\sum_{j=1}^{J} \nu_{i j} r_{j} \\
& r_{j}=k_{j}\left(\prod_{i=1}^{N}\left(\rho \frac{Y_{i}}{\overline{M_{i}}}\right)^{\nu_{i j}^{\prime}}-\frac{1}{K_{j}^{c}} \prod_{i=1}^{N}\left(\rho \frac{Y_{i}}{\overline{M_{i}}}\right)^{\nu_{i j}^{\prime \prime}}\right), \\
& k_{j}=a_{j} T^{\beta_{j}} \exp \left(\frac{-\bar{E}_{j}}{\mathcal{R} T}\right) \text {, } \\
& K_{j}^{c}=\left(\frac{p^{r e f}}{\mathcal{R} T}\right)^{\sum_{i=1}^{N} \nu_{i j}} \exp \left(\frac{-\sum_{i=1}^{N} \bar{g}_{i}^{o} \nu_{i j}}{\mathcal{R} T}\right), \\
& \sum_{i=1}^{N} Y_{i}=1 \\
& \sum_{i=1}^{N} \mathbf{j}_{i}=\mathbf{0} \\
& \sum_{i=1}^{N} \phi_{l i} \nu_{i j}=0 \\
& \nu_{i j}=\nu_{i j}^{\prime \prime}-\nu_{i j}^{\prime},
\end{aligned}
$$

where $\bar{M}$ is the mixture molecular mass, $D_{i k}$ the multi-component diffusion coefficient between the $i^{t h}$ and $k^{t h}$ species, $y_{i}$ the mole fraction of the $i^{t h}$ specie, $D_{i}^{T}$ the thermal diffusion coefficient of the $i^{t h}$ specie, $T$ the temperature, $\mu$ the dynamic viscosity of the mixture, $\mathrm{k}$ the thermal conductivity of the mixture, $R$ the mixture gas constant, $c_{P, i}$ the specific heat at constant pressure, $h_{i}$ the specific enthalpy, and $h_{i}^{r e f}$ the specific enthalpy evaluated at the reference pressure of the $i^{\text {th }}$ specie, $\mathcal{R}$ the universal gas constant which is $8.314 \times 10^{7} \mathrm{erg} /($ mole $K), p^{r e f}$ the reference pressure which is $1.01325 \times 10^{6} \mathrm{dyne}_{\mathrm{cm}} \mathrm{cm}^{\mathrm{ref}}$ the reference temperature which is $298.15 \mathrm{~K}, \nu_{i j}$ the net stoichiometric coefficient, $\nu_{i j}^{\prime}$ the forward stoichiometric coefficient, and $\nu_{i j}^{\prime \prime}$ the reverse stoichiometric coefficient of the $i^{\text {th }}$ specie in the $j^{\text {th }}$ reaction, $r_{j}$ the reaction rate, $k_{j}$ the Arrhenius model for the reactions' temperature sensitivity, $a_{j}$ the collision frequency factor, $\beta_{j}$ 
the temperature exponent, $\bar{E}_{j}$ the activation energy, and $K_{j}^{c}$ the equilibrium constant for the $j^{\text {th }}$ reaction, $\bar{g}_{i}^{o}$ the $i^{\text {th }}$ species' chemical potential at the reference pressure, and $\phi_{l i}$ the number of atoms of element $l$ in the $i^{t h}$ species. Equations (5)-(7) give constitutive relations for mass, momentum, and energy diffusion, which are an extended Fick's law, a Newtonian stress-strain rate relation, and an extended Fourier's law. The form of both Fourier's and Fick's law are appropriate for a mixture of ideal gases, as detailed in a derivation by Merk ${ }^{18}$ and summarized by Kee et al. ${ }^{19}$ and account for multicomponent mass diffusion as well as Soret and DuFour effects. Equation (6) has used Stokes' assumption. The mixture properties and reaction properties are evaluated using the Transport ${ }^{19}$ and CHEMKIN $^{20}$ packages, respectively. Note for inviscid calculations, all diffusion coefficients, viscosity, and thermal conductivity are taken to be zero.

\section{B. Computational method}

For inviscid calculations, the shock-fitting strategy of Henrick et al. ${ }^{17}$ was adapted to allow for an arbitrary number of chemical species as well as equations of state for ideal mixtures of calorically imperfect ideal gases, suitable for evaluation with the CHEMKIN package and enforces the shock state at the boundary. The underlying numerical scheme is a pointwise method of lines with fifth order spatial and fifth order RungeKutta temporal discretization. A central spatial scheme was used with special one-sided differences utilized near the shock. For the viscous calculations, the Wavelet Adaptive Multiresolution Representation (WAMR) method, first developed by Vasilyev and Paolucci, ${ }^{21,22}$ was used. WAMR is an adaptive mesh refinement technique using wavelets. These functions have compact support in both space and scale. This compact support allows for a large compression of data, enabling the use of many less points to accurately represent a flow field relative to a wide variety of other approaches. The WAMR method has been applied successfully to number problems in a serial computational environment. ${ }^{7,23-27}$ For a detailed description of the method in its current form, see Zikoski. ${ }^{28}$ Typical simulation times for the inviscid calculations were $\sim 2.5 \mu s /$ per day and for viscous calculations $\sim 1 \mu s /$ per day on an AMD $2.4 \mathrm{GHz}$ processor with $512 \mathrm{kB}$ cache. In the inviscid calculations a total of 75 days was necessary to simulate $200 \mu \mathrm{s}$. In the viscous calculations a total of 45 days was necessary to simulate $50 \mu s$.

\section{Results}

A series of inviscid and viscous calculations for one-dimensional, overdriven, hydrogen-air detonations was performed at ambient conditions of $293.15 \mathrm{~K}$ and $0.421 \mathrm{~atm}$ with the initial stoichiometric mixture of $2 \mathrm{H}_{2}+\mathrm{O}_{2}+3.76 \mathrm{~N}_{2}$. This choice of ambient conditions was made to enable direct comparison with previous calculations done by Daimon and Matsuo as well as the experiments of Lehr. ${ }^{29}$ It is also very close to conditions examined by Yungster and Radhakrishan. We employ the detailed kinetic mechanism used by Powers and Paolucci, drawn from Miller et al. ${ }^{30}$ shown in Table 1. which is composed of 19 reversible reactions, contains 9 species and 3 elements. Using this mechanism and these ambient conditions yields a $C J$ velocity of $D_{C J} \sim 1961 \mathrm{~m} / \mathrm{s}$, which is similar to that found by Sussman, $D_{C J} \sim 1958 \mathrm{~m} / \mathrm{s}$.

The use of continuum models like the Euler or Navier-Stokes equations is sometimes called into question at the fine scales needed in detonation modeling. The mean-free path is the cut-off minimum length scale associated with continuum theories. Using the simple definition given by Vincenti and Kruger, ${ }^{31}$ the meanfree path in a $f=1.150$ overdriven detonation is $\lambda=\bar{M} /\left(\sqrt{2} \pi \mathcal{N}_{A} \rho d^{2}\right) \approx 3 \times 10^{-6} \mathrm{~cm}$, where $\bar{M} \approx$ $20.911 \mathrm{~g} /$ mole, $\mathcal{N}_{A}=6.022 \times 10^{23}$ molecules $/$ mole is Avogadro's constant, $\rho \approx 2.00 \times 10^{-3} \mathrm{~g} / \mathrm{cm}^{3}$, and $d \approx$ $3.621 \times 10^{-8} \mathrm{~cm}$ is the molecular collision diameter. The overdrive of a detonation is defined as $f=D_{o}^{2} / D_{C J}^{2}$, where $D_{o}$ is the initial detonation velocity, and $D_{C J}$ is the Chapman-Jouguet velocity. Using an approximate kinematic viscosity in the burned gases of $\nu=6 \times 10^{-1} \mathrm{~cm}^{2} / \mathrm{s}$ and a frozen sound speed in the reacted zone of $c \approx 9 \times 10^{4} \mathrm{~cm} / \mathrm{s}$, an approximate viscous length scale is $\nu / c=6.67 \times 10^{-6} \mathrm{~cm} \approx \mathcal{O}\left(10^{-5} \mathrm{~cm}\right)$. The finest reaction length scale was calculated by the spatial eigenvalue analysis method of Powers and Paolucci and was found to be near $10^{-4} \mathrm{~cm}$. The finest reaction length scales are near those of the viscous scales, and the viscous terms in the Navier-Stokes equations may become important. The necessary discretizations to capture the inherent physics in the inviscid and viscous models are within the continuum regime. The Knudsen number, $K n=\lambda / L$, where $L$ is a representative length scale of the problem, is an indicator of how well continuum model captures the physics. Application of continuum models requires $K n \lesssim \mathcal{O}\left(10^{0}\right)$. The respective Knudsen numbers are $K n=3 \times 10^{-2}$ and $K n=4 \times 10^{-1}$ for the inviscid and viscous model physics. 
Table 1. Hydrogen-air reaction mechanism

\begin{tabular}{|c|c|c|c|c|}
\hline $\mathrm{j}$ & Reaction & $a_{j}\left(\left(\text { mole } / \mathrm{cm}^{3}\right)^{\left(1-\sum_{i=1}^{N} \nu_{i j}^{\prime}\right)} /\left(K^{\beta_{j}} s\right)\right)$ & $\beta_{j}$ & $E_{j}\left(\frac{c a l}{\text { mole }}\right)$ \\
\hline 1 & $\mathrm{H}_{2}+\mathrm{O}_{2} \rightleftharpoons 2 \mathrm{OH}$ & $1.70 \times 10^{13}$ & 0.00 & 47780 \\
\hline 2 & $\mathrm{OH}+\mathrm{H}_{2} \rightleftharpoons \mathrm{H}_{2} \mathrm{O}+\mathrm{H}$ & $1.17 \times 10^{9}$ & 1.30 & 3626 \\
\hline 3 & $H+\mathrm{O}_{2} \rightleftharpoons \mathrm{OH}+\mathrm{O}$ & $5.13 \times 10^{16}$ & -0.816 & 16507 \\
\hline 4 & $\mathrm{O}+\mathrm{H}_{2} \rightleftharpoons \mathrm{OH}+\mathrm{H}$ & $1.80 \times 10^{10}$ & 1.00 & 8826 \\
\hline 5 & $H+\mathrm{O}_{2}+M \rightleftharpoons \mathrm{HO}_{2}+M^{a}$ & $2.10 \times 10^{18}$ & -1.00 & 0 \\
\hline 6 & $H+\mathrm{O}_{2}+\mathrm{O}_{2} \rightleftharpoons \mathrm{HO}_{2}+\mathrm{O}_{2}$ & $6.70 \times 10^{19}$ & -1.42 & 0 \\
\hline 7 & $H+\mathrm{O}_{2}+\mathrm{N}_{2} \rightleftharpoons \mathrm{HO}_{2}+\mathrm{N}_{2}$ & $6.70 \times 10^{19}$ & -1.42 & 0 \\
\hline 8 & $\mathrm{OH}+\mathrm{HO}_{2} \rightleftharpoons \mathrm{H}_{2} \mathrm{O}+\mathrm{O}_{2}$ & $5.00 \times 10^{13}$ & 0.00 & 1000 \\
\hline 9 & $\mathrm{H}+\mathrm{HO}_{2} \rightleftharpoons 2 \mathrm{OH}$ & $2.50 \times 10^{14}$ & 0.00 & 1900 \\
\hline 10 & $\mathrm{O}+\mathrm{HO}_{2} \rightleftharpoons \mathrm{O}_{2}+\mathrm{OH}$ & $4.80 \times 10^{13}$ & 0.00 & 100 \\
\hline 11 & $2 \mathrm{OH} \rightleftharpoons \mathrm{O}+\mathrm{H}_{2} \mathrm{O}$ & $6.00 \times 10^{8}$ & 1.30 & 0 \\
\hline 12 & $H_{2}+M \rightleftharpoons H+H+M^{b}$ & $2.23 \times 10^{12}$ & 0.50 & 92600 \\
\hline 13 & $\mathrm{O}_{2}+M \rightleftharpoons O+O+M$ & $1.85 \times 10^{11}$ & 0.50 & 95560 \\
\hline 14 & $\mathrm{H}+\mathrm{OH}+\mathrm{M} \rightleftharpoons \mathrm{H}_{2} \mathrm{O}+M^{c}$ & $7.50 \times 10^{23}$ & -2.60 & 0 \\
\hline 15 & $\mathrm{H}+\mathrm{HO}_{2} \rightleftharpoons \mathrm{H}_{2}+\mathrm{O}_{2}$ & $2.50 \times 10^{13}$ & 0.00 & 700 \\
\hline 16 & $\mathrm{HO}_{2}+\mathrm{HO}_{2} \rightleftharpoons \mathrm{H}_{2} \mathrm{O}_{2}+\mathrm{O}_{2}$ & $2.00 \times 10^{12}$ & 0.00 & 0 \\
\hline 17 & $\mathrm{H}_{2} \mathrm{O}_{2}+\mathrm{M} \rightleftharpoons \mathrm{OH}+\mathrm{OH}+\mathrm{M}$ & $1.30 \times 10^{17}$ & 0.00 & 45500 \\
\hline 18 & $\mathrm{H}_{2} \mathrm{O}_{2}+\mathrm{H} \rightleftharpoons \mathrm{HO}_{2}+\mathrm{H}_{2}$ & $1.60 \times 10^{12}$ & 0.00 & 3800 \\
\hline 19 & $\mathrm{H}_{2} \mathrm{O}_{2}+\mathrm{OH} \rightleftharpoons \mathrm{H}_{2} \mathrm{O}+\mathrm{HO}_{2}$ & $1.00 \times 10^{13}$ & 0.00 & 1800 \\
\hline Enl & \multicolumn{4}{|c|}{$\begin{array}{l}M^{a}: f_{H_{2} O}=21.0, f_{H_{2}}=3.30, f_{N_{2}}=0.00, f_{O_{2}}=0.00 \\
M^{b}: f_{H_{2} O}=6.00, f_{H}=2.00, f_{H_{2}}=3.00 \\
M^{c}: f_{H_{2} O}=20.0\end{array}$} \\
\hline
\end{tabular}

\section{A. Inviscid overdriven detonations}

Time-dependent inviscid calculations were performed using a highly accurate shock-fitting technique to avoid issues associated with shock-capturing techniques when solving the Euler equations, e.g. spurious oscillations as seen by Quirk. ${ }^{32}$ These calculations were performed using a uniform spatial discretization of one micron in a shock-attached frame.

\section{Steady-structure of an overdriven detonation}

The steady-state structure was calculated in the manner of Powers and Paolucci, integrating only spatial ordinary differential equations using a fifth-order Runge-Kutta scheme with a spatial discretization of $10^{-8} \mathrm{~cm}$. The pressure profile occurring behind a $f=1.150$, overdriven hydrogen-air detonation is shown in Fig. 1(a). The pressure begins to decrease near $x=10^{-2} \mathrm{~cm}$, indicating the end of the induction zone. The evolution of species behind this same overdriven detonation front is shown in Fig. T(b). The disparity in the reaction length scales is clearly depicted. The first minor specie, $O H$, departs from power law growth near $x=4 \times 10^{-4} \mathrm{~cm}$. This indicates the beginning of the induction zone. Final relaxation of the species to equilibrium occurs near $x=10^{0} \mathrm{~cm}$. Thus, to accurately capture the true dynamics of the detonation, the minimum spatial discretization necessary is on the order of a micron, and the macroscale must be on the order of centimeters or longer.

\section{Stable detonation}

A prediction of the time-dependent behavior of the same $f=1.150$ overdriven detonation was initialized using a highly-resolved steady-state solution of the type presented in the previous section using a domain of $10 \mathrm{~cm}$. Figure 2 shows the computed detonation front pressure of the time-dependent prediction as well as the steady ZND detonation pressure. It is clear that the time-dependent prediction relaxes to the steady solution. The minute differences at long times between the time-dependent and steady-state predictions result from using a slightly more coarse spatial resolution in the time-dependent calculation. 


\section{Unstable detonations}

Using the shock-fitting technique, various overdrives were examined, and the neutral stability boundary was found at an overdrive of $f \approx 1.130$. Several of these overdriven inviscid detonation predictions are shown in Fig. 3. For overdrives of $1.025<f<1.130$ pulsations at times long relative to the oscillation period were predicted. As an example, for a $f=1.125$ overdriven detonation, oscillations with a frequency of $1.00 \mathrm{M} \mathrm{Hz}$ developed. For $f<1.025$, the pulsation amplitudes become large enough to cause the curve fits used for thermodynamic properties to be invalid.

In shock-induced combustion flow around spherical projectiles in hydrogen-air mixtures Lehr observed longitudinal oscillations. At an inflow condition corresponding to an overdrive of $f \approx 1.10$, Lehr observed a frequency of $1.04 \mathrm{MHz}$. For this same overdrive, we predict a frequency of $0.97 \mathrm{MHz}$. Similar results were reported by Yungster and Radhakrishan. As an example for an overdrive of $f=1.09$ with an ambient temperature of $298 \mathrm{~K}$ they found a frequency of $1.06 \mathrm{M} \mathrm{Hz}$. Thus, it seems that the instability observed by Lehr in multiple dimensions is captured well by a one-dimensional model. As the initial overdrive is lowered, the long time behavior of the inviscid detonations becomes more complex with the amplitude of the oscillations increasing and oscillations appearing at different frequencies. Daimon and Matsuo also found similar results. As example they found for a $f=1.10$ overdriven detonation a maximum detonation pressure of $14.0 \mathrm{~atm}$. For this same overdrive, we found the maximum detonation front pressure of $13.5 \mathrm{~atm}$. They do not report frequencies explicitly, but visual inspection suggests that the frequencies are in the $1 \mathrm{MHz}$ range. The neutral stability boundary found here is slightly lower than that found by Daimon and Matsuo. Though they do not explicitly state it, it can be inferred that their neutral stability boundary is closer to $f=1.20$. This discrepancy may be due to difference in chemical mechanisms used. We also speculate that the numerical diffusion and other issues associated shock capturing have an effect on the calculation of the stability boundary. Also, the initial numerical perturbations which incite the physical instability are many orders of magnitude smaller with this shock-fitting scheme compared with a shock-capturing scheme; thus, the closer to the neutral stability boundary, the longer the instability takes to reach a limit cycle.

\section{B. Viscous effects}

Using the WAMR, as described in Section [IB with an error tolerance of $\epsilon=5 \times 10^{-4}$, several simulations of viscous detonations were performed. These simulations were initialized using the ZND profile with a superimposed smooth transition from the shocked state to the ambient state over $10^{-3} \mathrm{~cm}$. The finest resolution utilized in these viscous simulations was $7 \times 10^{-6} \mathrm{~cm}$.

Examining a viscous detonation with an overdrive above the inviscid neutral stability point, it was found


pressure versus time in both the viscous and inviscid cases. The early time perturbations present in the viscous case are due to the use of the ZND profile for initialization. However, by about $40 \mu s$, the initial perturbations of the detonation front have nearly relaxed to zero. Even though both cases exhibit stability, Figs. 5 and 6 show that, while small, viscous effects still affect the shape of the detonation front. Figure 5 shows the long time structure of the $O H$ mass fraction, and it is clear that the viscous effects cause a slight lengthening of the induction zone. It can be discerned from Fig. 6 that in the stable case viscous processes only have a slight effect on the structure of pressure, smoothing the detonation front.

Examining a lower overdrive, $f=1.100$, an unstable detonation is predicted in the inviscid limit. Figure 7 shows that the viscous equivalent is also unstable; however, with the addition of viscous effects, the amplitude of the oscillations is damped by $\sim 25 \%$. Moreover, we anticipate by analogy with our one-step results ${ }^{16}$ that more extensive calculations will reveal that the stability boundary is delayed by the inclusion of viscous effects, and in the highly unstable regime the effects of viscosity on the long time dynamics will be large. Figure 8 shows the structure of the pressure wave at $t=40 \mu \mathrm{s}$ for the viscous and $t=140 \mu \mathrm{s}$ inviscid case. In both cases, the pressure wave trailing the detonation front shows oscillations which are manifestations of the detonation pressure oscillations. However, in the zoomed portion of Fig. 8 it is clear that the detonation peak is spread by the addition of viscous effects.

\section{Conclusions}

An investigation of one-dimensional, unsteady, overdriven, hydrogen-air detonations has shown, consistent with previous studies by Sussman, Yungster and Radhakrishan, and Daimon and Matsuo, that as the 
initial overdrive is decreased, the long time behavior becomes progressively more complex. At high overdrives, the detonations are stable. At a critical overdrive, $f \approx 1.130$, for a detonation propagating into a stoichiometric hydrogen-air mixture at $0.421 \mathrm{~atm}$ and $293.15 \mathrm{~K}$, single frequency pulsations appear. The amplitude of these pulsations increases as the overdrive is lowered further. As the overdrive is decreased even more, a bifurcation process ensues in which modes at a variety of frequencies are excited. In addition, the predicted $0.97 \mathrm{MHz}$ frequency for a $f=1.10$ overdriven detonation agrees well with the frequency of $1.04 \mathrm{MHz}$ observed by Lehr in his experiments of shock-induced combustion flow around spherical projectiles in a hydrogen-air mixture at a inflow corresponding to a similar overdrive. It has also been seen that the inclusion of mass, momentum, and energy diffusion modulates the structure of the overdriven detonation relative to the inviscid limit. Instead of a discrete jump, the detonation front becomes steep, yet smooth in the presence of viscous effects. Moreover, the addition of physical diffusion has a stabilizing effect on the long time behavior of a hydrogen-air overdriven detonation. Specifically, the amplitude of the oscillations is significantly decreased, and we speculate that further calculation will reveal viscosity to delay the onset of instability. This is the logical extension of the conclusions reached using the one-step kinetics model. ${ }^{16}$

\section{Acknowledgments}

This work was performed under support of the US Department of Energy.

\section{References}

${ }^{1}$ J. M. Powers and S. Paolucci. Accurate spatial resolution estimates for reactive supersonic flow with detailed chemistry. AIAA Journal, 43(5):1088-1099, 2005.

${ }^{2}$ A. N. Al-Khateeb, J. M. Powers, and S. Paolucci. On the necessary grid resolution for verified calculation of premixed laminar flames. Communications in Computational Physics, 8(2):304-326, 2010.

${ }^{3}$ J. E. Shepherd. Detonation in gases. Proceedings of the Combustion Institute, 32:83-98, 2009.

${ }^{4}$ M .A. Sussman. Numerical Simulation of Shock-Induced Combustion. PhD thesis, Stanford University, 1995.

${ }^{5}$ E. S. Oran, J. W. Weber, E. I. Stefaniw, M. H. Lefebvre, and J. D. Anderson. A numerical study of a two-dimensional $\mathrm{H}_{2}-\mathrm{O}_{2}-\mathrm{Ar}$ detonation using a detailed chemical reaction model. Combustion and Flame, 113:147-163, 1998.

${ }^{6}$ C. A. Eckett. Numerical and Analytical Studies of the Dynamics of Gaseous Detonations. PhD thesis, California Institute of Technology, 2001.

${ }^{7}$ S. Singh, Y. Rastigejev, S. Paolucci, and J. M. Powers. Viscous detonation in $H_{2}-O_{2}-A r$ using intrinsic lowdimensional manifolds and wavelet adaptive multilevel representation. Combustion Theory and Modelling, 5(2):163-184, 2001.

${ }^{8}$ S. Yungster and K. Radhakrishan. Pulsating one-dimensional detonations in hydrogen-air mixtures. Combustion Theory and Modelling, 8(4):745-770, 2004.

${ }^{9}$ X. Y. Hu, B. C. Khoo, D. L. Zhang, and Z. L. Jiang. The cellular structure of a two-dimensional $H_{2} / O_{2} / A r$ detonation wave. Combustion Theory and Modelling, 8(2):339-359, 2004.

${ }^{10}$ X. Y. Hu, D. L. Zhang, B. C. Khoo, and Z. L. Jiang. The structure and evolution of a two-dimensional $H_{2} / O_{2} / A r$ cellular detonation. Combustion Theory and Modelling, 14:37-44, 2004.

${ }^{11}$ Y. Daimon and A. Matsuo. Unsteady features on one-dimensional hydrogen-air detonations. Physics of Fluids, 19(11), 2007.

${ }^{12}$ J. L. Ziegler, R. Deiterding, J. E. Shepherd, and D. I. Pullin. An adaptive high-order hybrid scheme for compressive, viscous flows with detailed chemistry. Journal of Computational Physics, 230(20):7598-7630, 2011.

${ }^{13}$ N. Tsuboi, K. Eto, and A. K. Hayashi. Detailed structure of spinning detonation in a circular tube. Combustion and Flame, 149:144-161, 2007.

${ }^{14} \mathrm{R}$. Deiterding. A parallel adaptive method for simulating shock-induced combustion with detailed chemical kinetics in complex domains. Computers and Structures, 87:769-783, 2009.

${ }^{15}$ J. M. Powers. Review of multiscale modeling of detonation. Journal of Propulsion and Power, 22(6):1217-1229, 2006.

${ }^{16}$ C. M. Romick, T. D. Aslam, and J. M. Powers. The dynamics of unsteady detonation with diffusion. 49th AIAA Aerospace Sciences Meeting and Exhibit, Orlando, Florida, 2011. AIAA-2011-0799.

${ }^{17}$ A. K. Henrick, T. D. Aslam, and J. M. Powers. Simulations of pulsating one-dimensional detonations with true fifth order accuracy. Journal of Computational Physics, 213(1):311-329, 2006.

${ }^{18}$ H. J. Merk. The macroscopic equations for simultaneous heat and mass transfer in isotropic, continuous and closed systems. Applied Scientific Research. 8:73-99,1959.

${ }^{19}$ R. J. Kee, G. Dixon-Lewis, J. Warnatz, M. E. Coltrin, and J. A. Miller. A Fortran computer code package for the evaluation of gas-phase multi-component transport properties. Technical Report SAND86-8246, Sandia National Laboratories, 1991.

${ }^{20}$ R. J. Kee, F. M. Rupley, and J. A. Miller. Chemkin II: A Fortran chemical kinetics package for the analysis of gas phase chemical kinetics. Technical Report SAND89-8009B, Sandia National Laboratories, 1992.

${ }^{21}$ O. V. Vasilyev and S. Paolucci. Dynamically adaptive multilevel wavelet collocation method for solving partial differential equations in a finite domain. Journal of Computational Physics, 125:498-512, 1996. 
${ }^{22}$ O. V. Vasilyev and S. Paolucci. A fast adaptive wavelet collocation algorithm for multidimensional PDEs. Journal of Computational Physics, 138:16-56, 1997. 2002.

${ }^{23}$ Y. A. Rastigejev. Multiscale Computations with a Wavelet Adaptive Algorithm. PhD thesis, University of Notre Dame,

${ }^{24}$ Y. A. Rastigejev and S. Paolucci. Wavelet-based adaptive multiresolution computation of viscous reactive flows. International Journal for Numerical Methods in Fluids, 5:749-784, 2006.

${ }^{25}$ Y. A. Rastigejev and S. Paolucci. Wavelet adaptive multiresolution representation: Applications to viscous multiscale flow simulations. International Journal of Wavelets, Multiresolution, and Information Processing, 4:333-343, 2006.

${ }^{26} \mathrm{D}$. Wirasaet and S. Paolucci. An adaptive wavelet method for incompressible flows in complex domains. Journal of Fluids Engineering, 127:656-665, 2005.

${ }^{27}$ D. Wirasaet. Numerical Solutions of Multidimensional Partial Differential Equations Using an Adaptive Wavelet Method. PhD thesis, University of Notre Dame, 2008.

${ }^{28}$ Z. J. Zikoski. A Parallel Adaptive Wavelet Method for Multidimensional Simulations of Hypersonic Propulsion. PhD thesis, University of Notre Dame, 2011.

${ }^{29}$ H. F. Lehr. Experiments on shock-induced combustion. Astronautica Acta, 17:589-597, 1972.

${ }^{30}$ J. A. Miller, R. E. Mitchell, M. D. Smooke, and R. J. Kee. Toward a comprehensive chemical kinetic mechanism for the oxidation of acetylene: Comparison of model predictions with results from flame and shock tube experiments. In Proceedings of the Nineteenth Symposium (International) on Combustion, Combustion Institute, pages 181-196, 1982.

${ }^{31}$ W. G. Vincenti and C. H. Kruger. Introduction to Physical Gas Dynamics. Wiley, 1967.

${ }^{32}$ J. J. Quirk. A contribution to the great Riemann solver debate. International Journal for Numerical Methods in Fluids, 18:555-574, 1994.
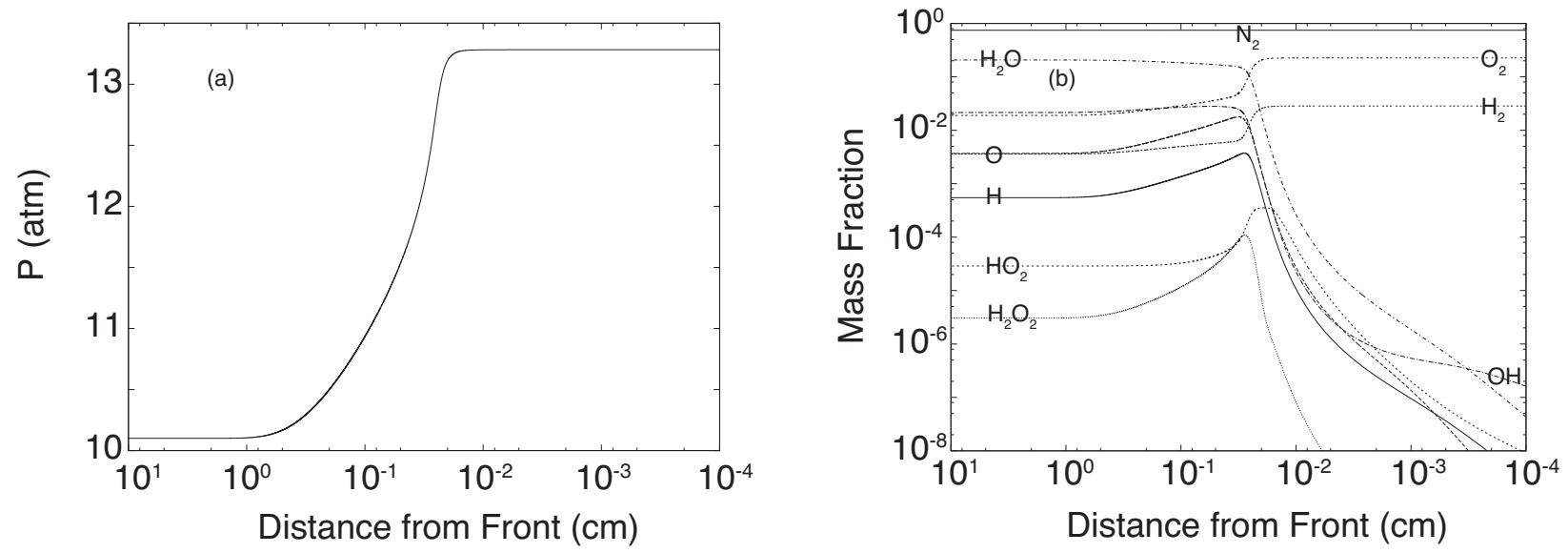

Figure 1: Structure of a steady inviscid, overdriven, hydrogen-air detonation, $f=1.150$ for (a) pressure and (b) mass fraction. The detonation front is traveling to the right.

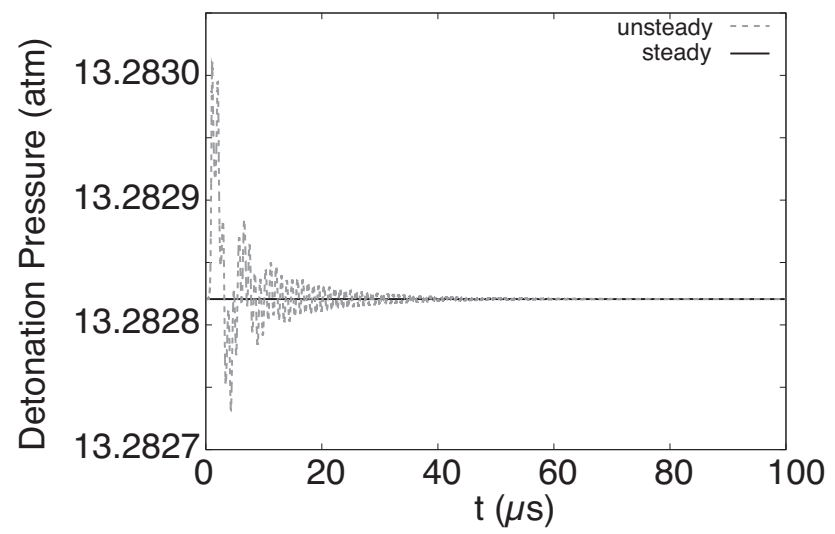

Figure 2: Time-dependent behavior for an inviscid, overdriven, $f=1.15$, hydrogen-air detonation with an ambient state of $0.421 \mathrm{~atm}$ and $293.15 \mathrm{~K}$ predicted using a shock-fitting technique. 

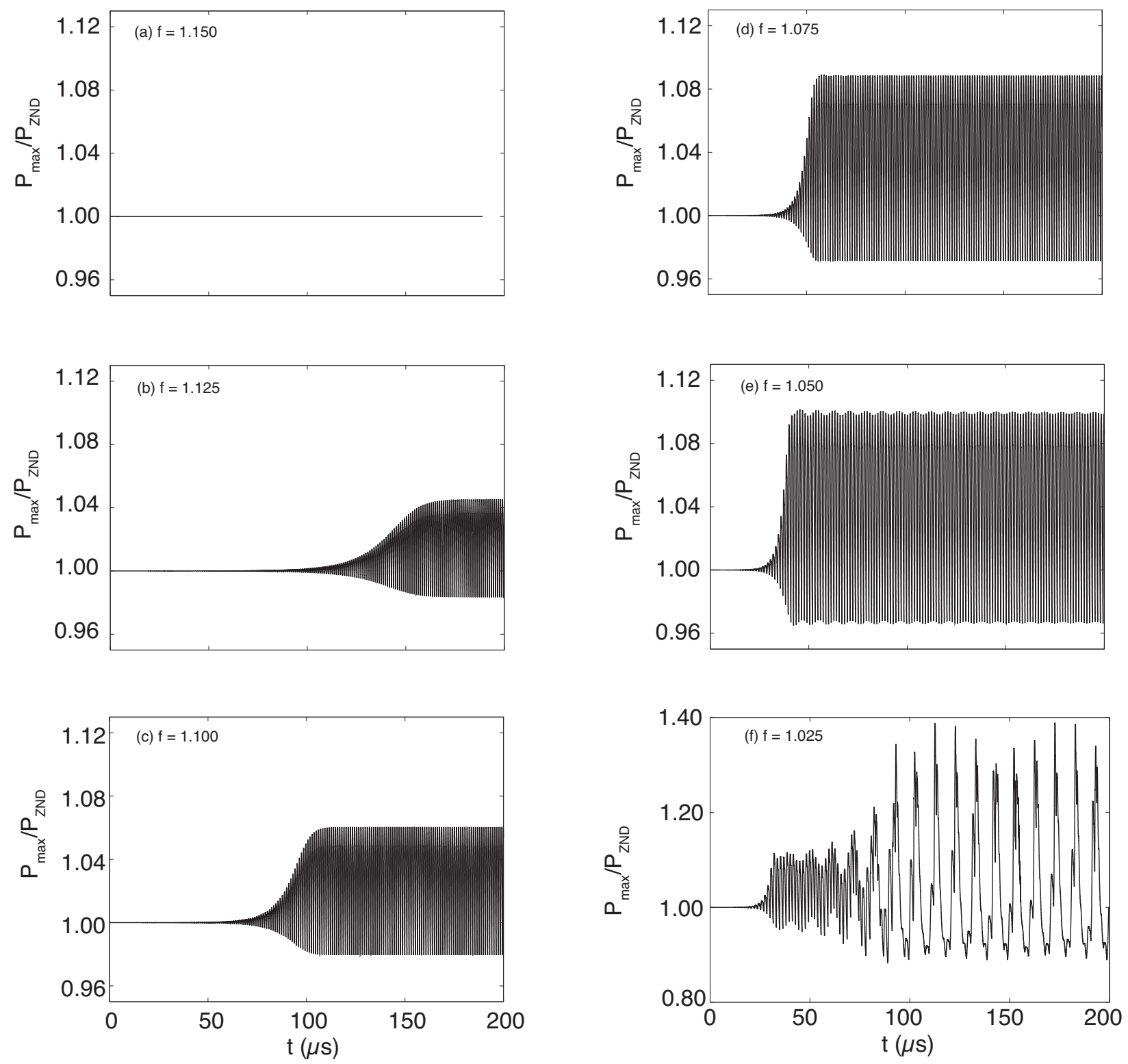

Figure 3: Time-dependent behavior for various inviscid, overdriven, hydrogen-air detonations with an ambient state of $0.421 \mathrm{~atm}$ and $293.15 \mathrm{~K}$ predicted using a shock-fitting technique. Initial overdrive decreases from (a) through (f). 


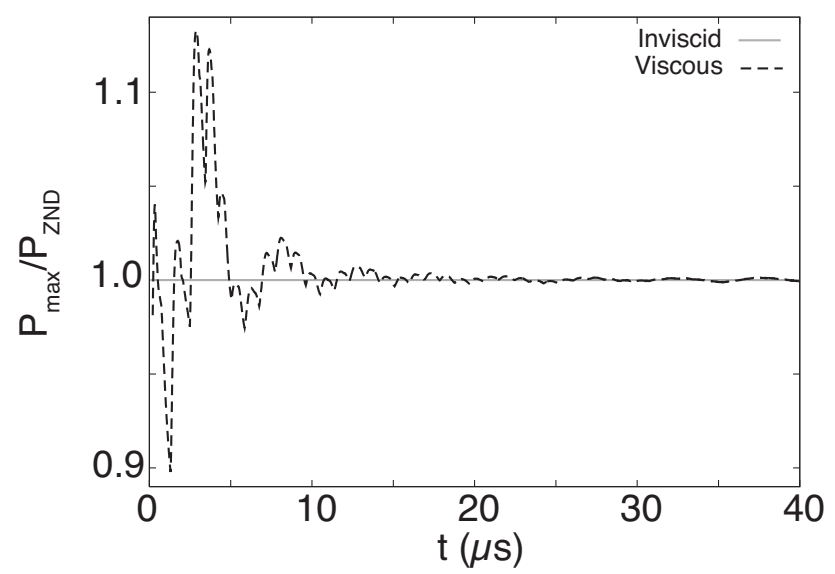

Figure 4: Time-dependent behavior for an overdriven, $f=1.150$, hydrogen-air detonation with an ambient state of $0.421 \mathrm{~atm}$ and $293.15 \mathrm{~K}$ for both the viscous and inviscid cases.

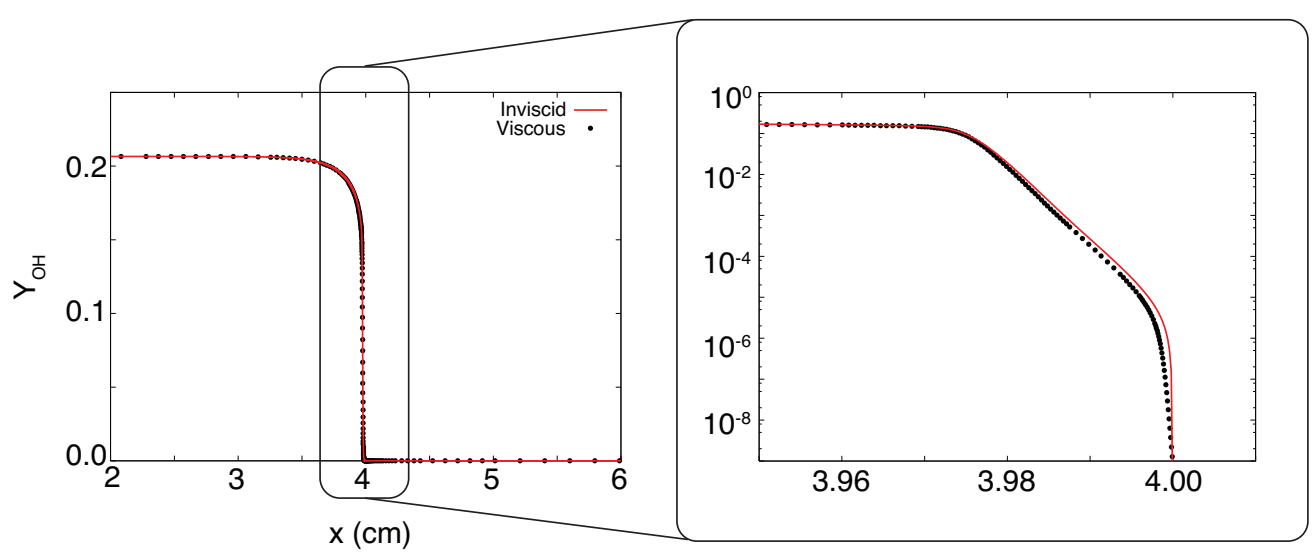

Figure 5: Structure of $O H$ mass fraction in an overdriven, $f=1.150$, hydrogen-air detonation with an ambient state of $0.421 \mathrm{~atm}$ and $293.15 \mathrm{~K}$ at $t=100 \mu \mathrm{s}$.

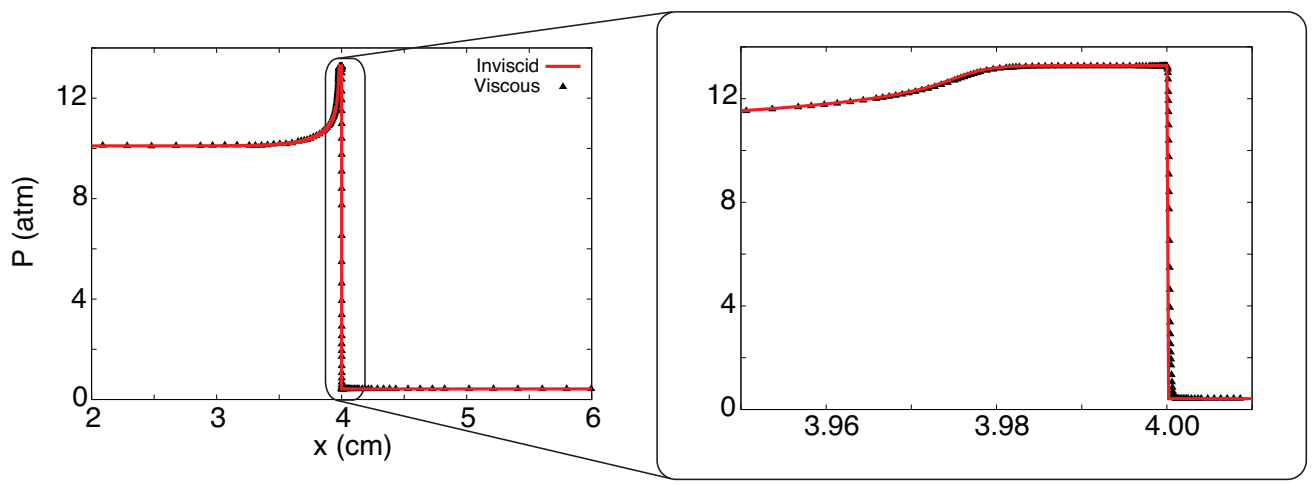

Figure 6: Structure of pressure in an overdriven, $f=1.150$, hydrogen-air detonation with an ambient state of $0.421 \mathrm{~atm}$ and $293.15 \mathrm{~K}$ at $t=100 \mu \mathrm{s}$. 


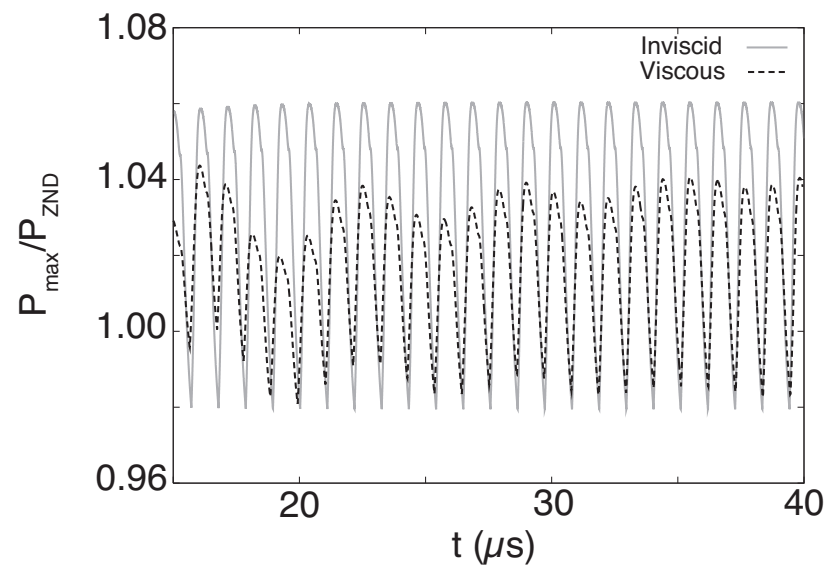

Figure 7: Time-dependent behavior for an overdriven, $f=1.100$, hydrogen-air detonation with an ambient state of $0.421 \mathrm{~atm}$ and $293.15 \mathrm{~K}$, for both the viscous and inviscid cases. The inviscid case has shifted in time by a $100 \mu s$.

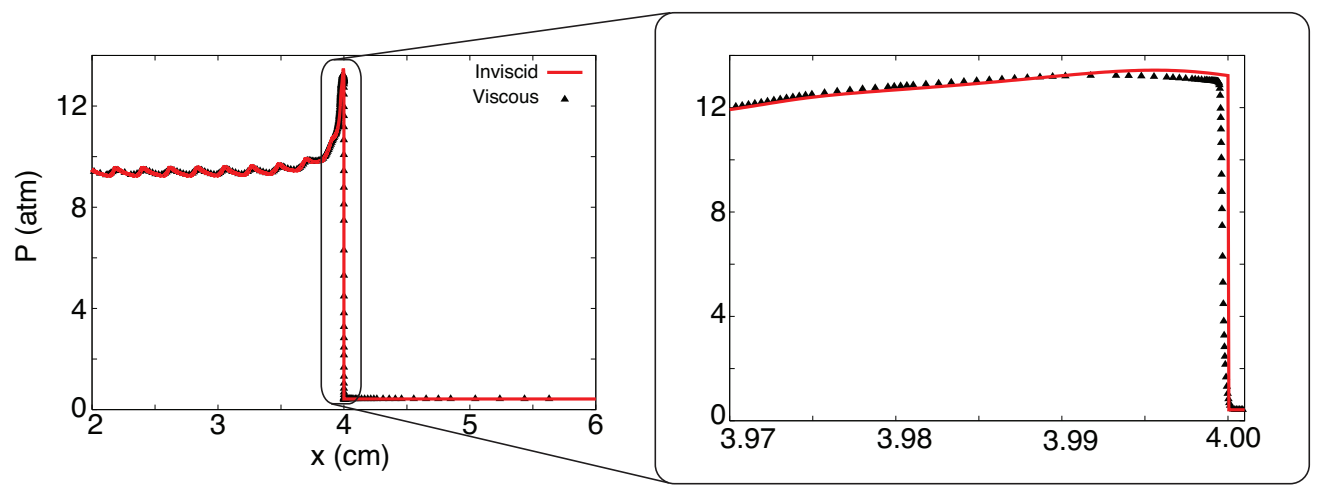

Figure 8: Structure of pressure in an overdriven, $f=1.100$, hydrogen-air detonation with an ambient state of $0.421 \mathrm{~atm}$ and $293.15 \mathrm{~K}$. The viscous case is shown at $t=40 \mu \mathrm{s}$, and the inviscid case is shown at $t=140 \mu s$. 